\title{
Edge and Line detection in polarimetric SAR images
}

\author{
D. Borghys \\ V. Lacroix \\ C. Perneel \\ Royal Military Academy, Signal \& Image Centre \\ Av. de la Renaissance 30, B-1000 Brussels \\ Dirk.Borghys@elec.rma.ac.be,Vinciane.Lacroix@elec.rma.ac.be, Christiaan.Perneel@twma.rma.ac.be
}

\begin{abstract}
A new scheme for detecting edges and lines in multichannel SAR images is proposed. The line detector is constructed from the edge detector. The latter is based on multivariate statistical hypothesis tests applied to log-intensity SAR images. The raw results are vectorized by a traditional bright line extraction process. The scheme is illustrated by extracting dark linear structures on various fullpolarimetric SAR images.
\end{abstract}

\section{Introduction}

Because of their independence of weather and lighting conditions, and their complementarity to visual and infrared sensors, synthetic aperture radar (SAR) images have been more and more used in the last few years. The increasing number of SAR systems used in remote sensing creates the need for (semi-)automatic tools for the interpretation of such images. In general, traditional image processing algorithms fail in SAR images because of an interference phenomenon, called speckle, which results in very noisy appearance of the images. Specific SAR image processing methods, which take into account the properties of the speckle, have been developed since the eighties. Existing methods are focussed on multi-look, mono-polarisation, single-frequency images (e.g. [9, 4] for line detection). Current airborne SAR systems and future satellite systems acquire multi-channel SAR data, i.e. full-polarmetric and/or multi-frequency data, because such data contain a lot more information.

In this paper, we present a method to extract edges and linear structures in multi-channel SAR images. First, an edge or line operator, based on multi-variate statistics, is applied to the image. These operators are respectively described in section 2 and 3 . Then, the raw results are converted into vectorised objects using a traditional line extraction process described in section 4 . In section 5, the approach is illustrated by the detection of dark linear struc- tures on full-polarimetric SAR images originating from different sensors. The originality of the method resides in the use of the multi-variate hypothesis tests and in the postprocessing proposed.

\section{Edge detection}

\subsection{In mono-polarisation $S A R$ images}

The presence of speckle makes pixel-wise methods using a simple filtering mask inappropriate for edge detection in SAR images. The solution that is commonly adopted is to take into account larger neighbourhoods of each pixel for deciding whether an edge passes through that pixel.

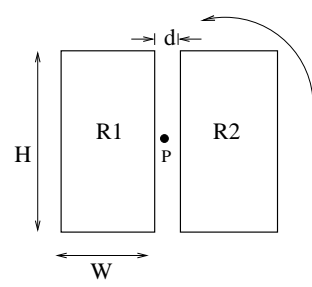

(a)

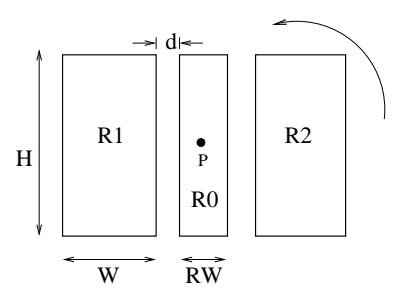

(b)

\section{Figure 1. Principle of edge (a) and line detec- tion (b) in SAR images}

The following principle is used (see Fig. 1a): the images are scanned by a set of two adjacent rectangular windows and in each pixel, local measurements, which are a function of statistical differences between the pixels in both windows, are used as an indication that the edge between the two windows actually corresponds to an edge in the image. This gives an answer for one possible edge orientation. The set of rectangles is rotated around the current pixel to verify the presence of an edge along other orientations. Typically 4 or 8 different orientations are tested and the maximum response is kept [3]. Existing methods were mostly applied on single-channel multi-look intensity images and differ by the used comparison criterion [3]. The most well-known is 
the ratio detector [7] which considers the ratio of average intensities between the two rectangles.

\subsection{In polarimetric $S A R$ images}

A polarimetric SAR image provides a measurement of the backscattering matrix in each pixel of the image, i.e. each pixel is characterised by a value for each of the three polarisations $\mathrm{HH}, \mathrm{HV}$ and $\mathrm{VV}$. An obvious way to detect edges in such multi-channel images is to fuse the results of existing detectors applied on each individual channel. An alternative is to use multi-variate statistical methods which treat the combined information from the different channels as a single input-vector.
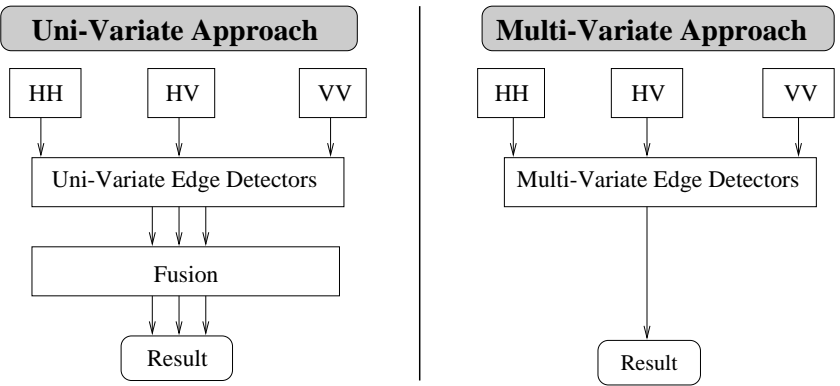

Figure 2. Edge Detection in Polarimetric SAR

Fig. 2 illustrates the two approaches that can be used for detecting edges in multi-channel SAR images. For edge detection in full-polarimetric SAR images, single-look, logintensity SAR images we introduced a Hotellings- $T^{2}$ test in [2]. This test for the difference of means is defined as:

$$
T^{2}=\frac{n_{1} n_{2}\left(\overline{\mathbf{X}_{\mathbf{1}}}-\overline{\mathbf{X}_{\mathbf{2}}}\right)^{t} \mathbf{C}^{-1}\left(\overline{\mathbf{X}_{\mathbf{1}}}-\overline{\mathbf{X}_{\mathbf{2}}}\right)}{n_{1}+n_{2}}
$$

whit $n_{1}$ and $n_{2}$ the number of observations and $\overline{\mathbf{X}_{\mathbf{k}}}$ the average vector of the observations in the two scanning rectangles. $\mathbf{C}$ is the pooled covariance matrix. In the nullhypothesis of equal population means we have:

$$
T_{F}=\frac{\left(n_{1}+n_{2}-p-1\right) T^{2}}{\left(n_{1}+n_{2}-2\right) p} \sim F\left(p, n_{1}+n_{2}-p-1\right),
$$

whit $p$ the number of images and $F$ the Fisher-Snedecor distribution. The results obtained by the multi-variate method are better than those obtained by fusing the outcomes of uni-variate detectors [1]. The reason is that multi-variate methods use the covariance matrix of the dataset and thus take into account not only the intensity but also the correlation between the different data-channels.

\section{Line detection}

In $[8,9]$ a method for constructing a line detector from edge detectors is presented for SAR images. This time three rectangles are used. The middle one is centered on the current point and is narrower than the two others (see fig. 1b). In order to detect whether a line in a given orientation passes through the point $P$, the statistics of the two outer rectangles are compared with those in the middle one. The response of the detector is the response corresponding to the smallest difference in statistics. This allows to avoid false alarms due to (single) edges. The width of the central rectangle is chosen such that it corresponds approximatively to the possible widths of the lines (e.g. roads) to be detected. We apply this method to construct a line detector from our multi-variate edge detector.

In SAR images it is often possible to predict whether a given object will appear as a bright or a dark line. Roads for instance will almost always appear as dark lines because their surface is very smooth compared to the wavelength of the radar. In this case an extra condition can be applied in the line detection algorithm. The condition is based on a comparison of the average image intensity of the centre rectangle $A_{R_{o}}$ with the one found in the two outer rectangles $A_{R_{1}}$ and $A_{R_{2}}$. The response $F_{T}$ of a detector for dark lines is then:

$F_{T}= \begin{cases}\min \left(F_{R_{o}, R_{1}}, F_{R_{o}, R_{2}}\right) & \text { if }\left(A_{R_{o}}<A_{R_{1}}\right) \operatorname{and}\left(A_{R_{o}}<A_{R_{2}}\right) \\ 0 & \text { otherwise }\end{cases}$

where $F_{R_{o}, R_{i}}$ is the response of the edge detector between rectangles $R_{o}$ and $R_{i}$.

\section{Post-Processing}

Usually, a nonmaximum suppression in the direction perpendicular to the local feature provides one pixel wide elements that are linked to form a vectorial representation. Despite the fact that bright lines are clearly seen on Figure 3 displaying the SAR operator output, this algorithm provides multiple responses because the lines are not smooth.
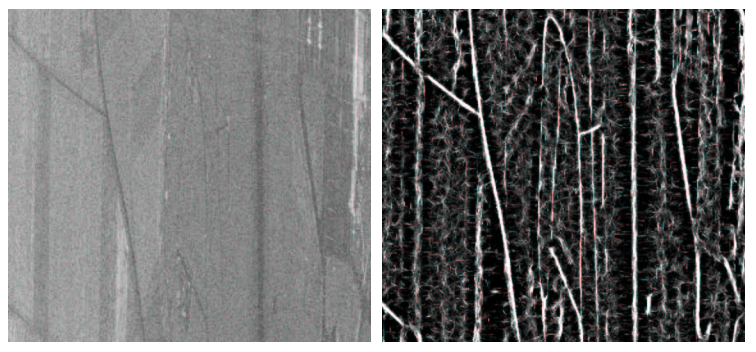

Figure 3. Example of line operator output

Several post-processing methods have been proposed to overcome these problems $[4,9]$. In this communication, we propose to apply a traditional line detection, like the Gradient Line Detector [5] (GLD), on the SAR operator output. The GLD involves three modules: a line operator, an extractor of maxima and a line following. The line operator 
exploits the fact that the gradient of the intensity is pointing in opposite direction at each side of a line. Thus, a gaussian gradient is first performed on the image; then, at each pixel, the dot product of the gaussian gradient at each pair of opposing pixels in the local neighborhood is computed and the maximum is taken as line value. The operator has two parameters: the color of the line to detect (bright or dark) and the $\sigma$ of the smoothing gaussian. The second module is a non-maximum suppression in the direction perpendicular to the line. Finally, the line following has three parameters: an upper line threshold $u$ at which a line could be started, a lower line threshold $l$ at which a line can be continued, and a line length $s$ threshold. Figure 4 illustrates the whole process.

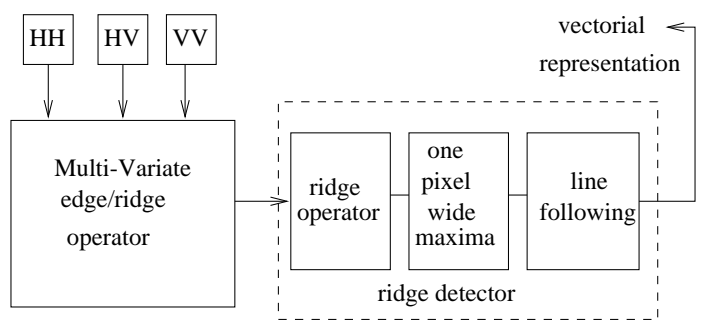

\section{Figure 4. edge/line extraction scheme for multi-channel SAR images}

\section{Results and discussion}

The proposed edge/line detection scheme is applied on two full-polarimetric airborne SAR images (an ESAR Lband and a PI-SAR X-band image) on which dark linear structures are sought. For this purpose, the line operator searching for dark lines is used with the following parameters: $\mathrm{RW}=2, \mathrm{~W}=10, \mathrm{H}=30, \mathrm{~d}=2$ and 16 orientations of the scanning windows.

Results are shown on parts of these images (see Figure 5); The VV polarization of a $867 \times 794$ region of the E-SAR image is displayed in (a). The GLD was used with $\sigma=1$, and $s=35$; the resulting objects are shown in (b). The objects were not added to the image for clarity reasons. A $1024 \times 1024$ region of the PI-SAR X-band VVpolarisation image is shown in (c). The vectorial results obtained by the uni-variate method of F. Tupin [9] and those obtained by our multi-variate method are shown in (e) and (f) respectively. The GLD was used with $\sigma=1, u=10$, $l=1, s=70$. In (d) part of a map is shown as a reference. Making a detailed comparison between these results is difficult for several reasons. Our method uses the three polarisations in a multi-variate approach while Tupin's method only uses the polarisation with which the roads are best visible (VV). For each algorithm, parameters need to be set which influence the results considerably. Neverthe- less it is possible to draw some conclusions: both methods give false alarms in densely built-up areas. Our method detects some shadows of forests as road candidates. We detect more roads without introducing too many false alarms. The method of Tupin includes a line completion step enabling the algorithm to bridge small gaps between linear segments. This can be seen on the river on the centre top of the image where her result follows the river over the bridge while our method links the river to the road to the right. Her method does not detect this road near the bridge. For enhancing our results, higher-level tools should be introduced in the postprocessing. This is a topic for further work. In particular a combination of our method with an MRF-based method as in [9], but adapted to multi-channel data, will be investigated.

\section{Acknowledgments}

The Institute of Radio Frequency Technology and Radarsystems of the German Aerospace Center (DLR) and the Japanese CRL/NASDA respectively provided the first and second test images. Special thanks to Florence Tupin (ENST, Paris) who processed our data in order to have a basis of comparison for the proposed scheme.

\section{References}

[1] D. Borghys. Interpretation and Registration of High-Resolution Polarimetric SAR Images. PhD thesis, ENST E 031, Paris, Nov 2001.

[2] D. Borghys, C. Perneel, and M. Acheroy. Contour detection in high-resolution polarimetric sar images. In SPIE Conference on SAR Image Analysis,Modelling and Techniques III; Barcelona, sept 2000.

[3] R. Fjörtoft. Segmentation d'images radar par detection de contour. PhD thesis, Institut National Polytechnique de Toulouse, Toulouse, March 1999.

[4] O. Hellwich, H. Mayer, and G. Winkler. Detection of lines sar scenes. In Proc. ISPRS Workshop, volume 31, pages 312320, Vienna, Austria, 1996.

[5] V. Lacroix and M. Acheroy. Feature-extraction using the constrained gradient. ISPRS Journal of Photogrammetry and Remote Sensing, 53(2):85-94, April 1998.

[6] B. Manly, editor. Multivariate Statistical Methods. Chapman and Hall, 1995.

[7] R. Touzi, A. Lopes, and P. Bousquet. A statistical and geometrical edge detector for sar images. IEEE-GRS, 26(6):764773, November 1988.

[8] F. Tupin. Reconnaissance des Formes et Analyse de Scènes en Imagerie Radar à Ouverture Synthétique. PhD thesis, ENST, Paris, September 1997.

[9] F. Tupin, H. Maître, J. Mangin, J. Nicolas, and E. Pechersky. Detection of linear features in sar images: Application to road network extraction. IEEE-GRS, 36(2):434-453, March 1998. 


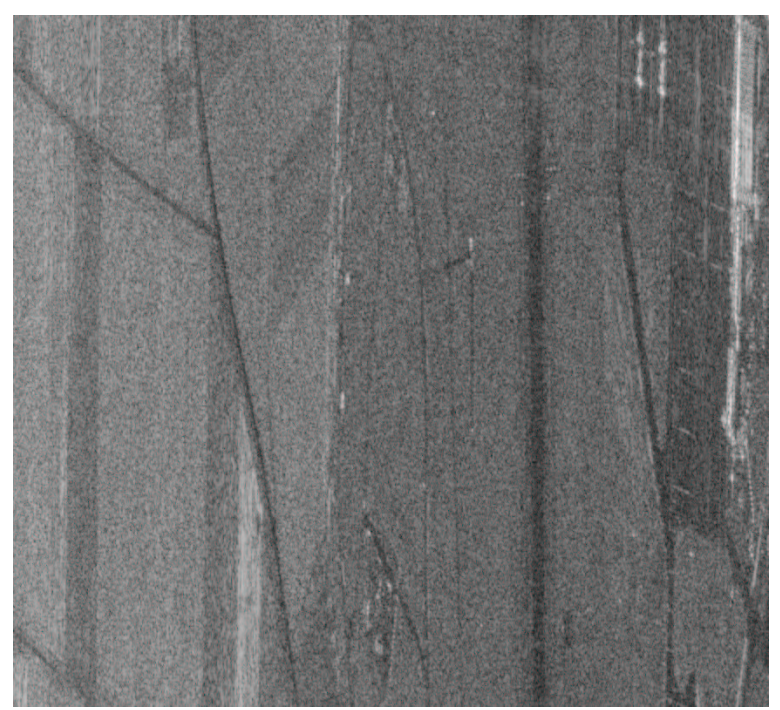

(a)

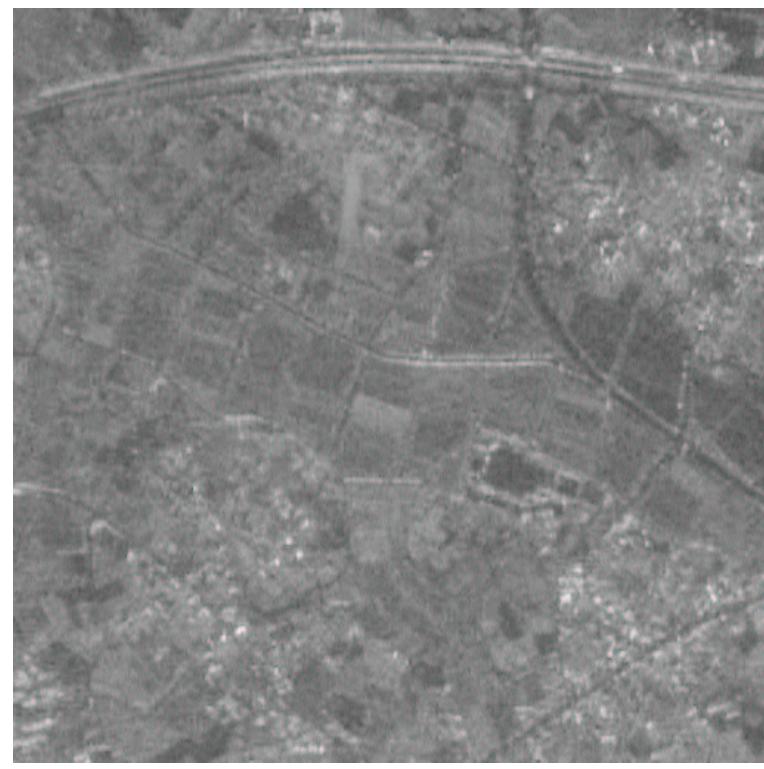

(c)

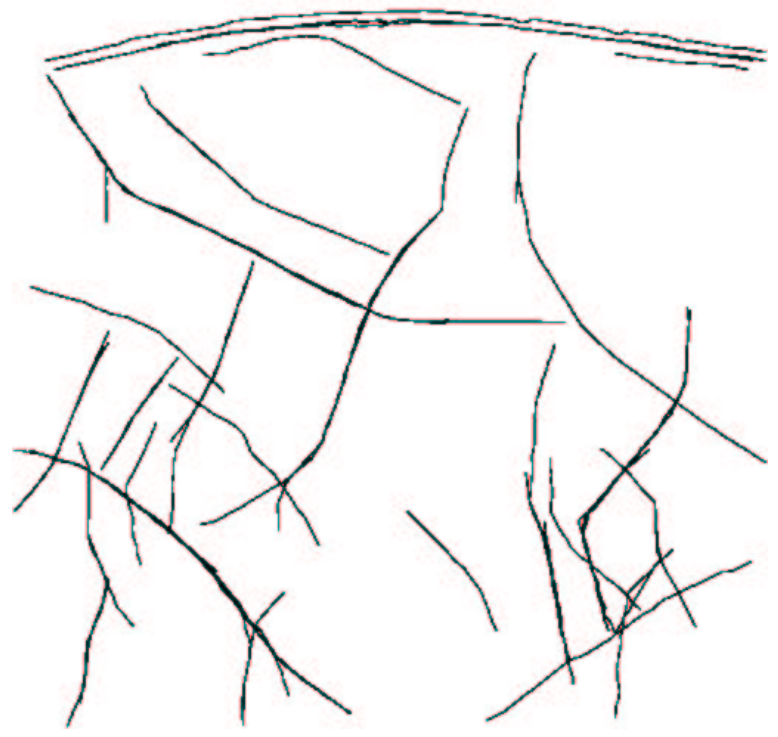

(e)

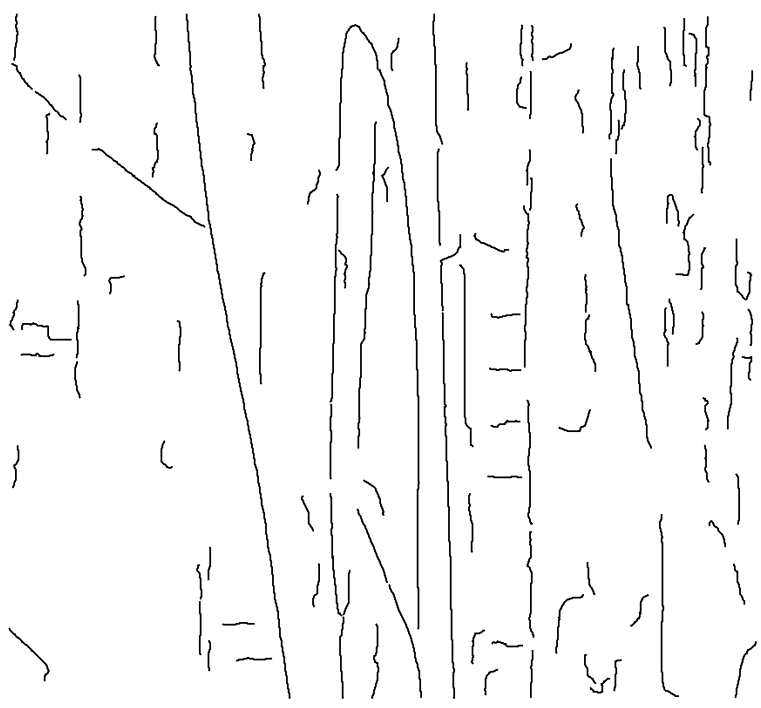

(b)

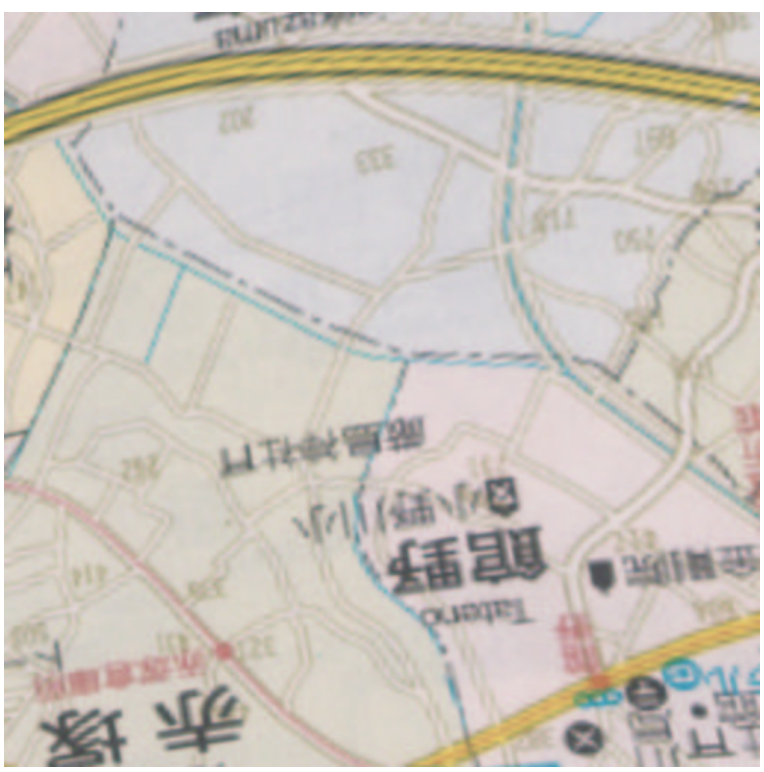

(d)

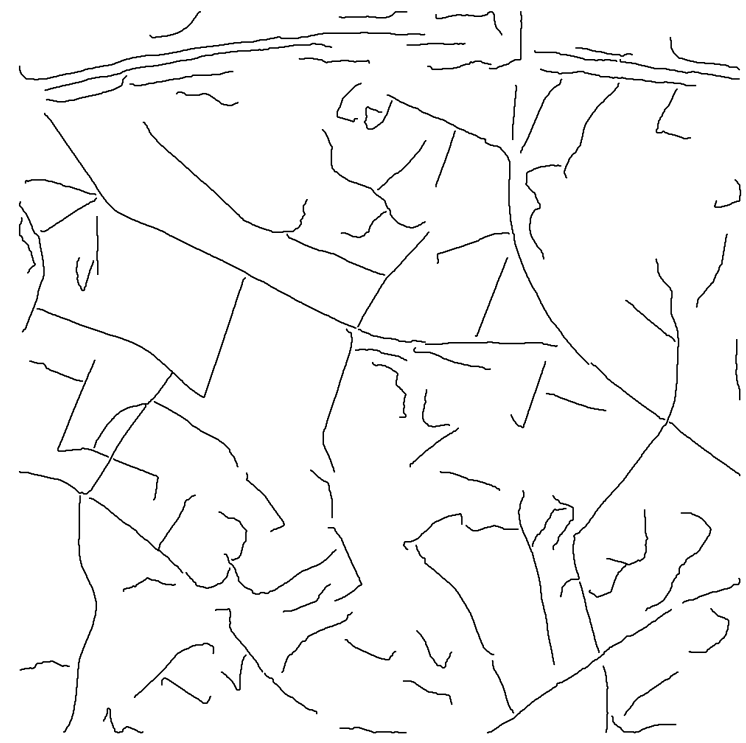

(f)

Figure 5. (a) First original SAR image $867 \times 794$ (VV); (b) Vectorization of the SAR detector using the GLD; (c) Second original SAR image $512 \times 512$ (VV); (d) Corresponding map; (e) Results of Tupin's method; (f) Results of our method. 